\title{
LA INCLUSIÓN DE LA "CUESTIÓN ECOLÓGICA» EN EL CURRÍCULO DE LOS/AS GRADUADOS/AS EN RELACIONES LABORALES Y RECURSOS HUMANOS
}

\author{
IgNASi LERMA MONTERo \\ Instituto Universitario de Investigación Interdisciplinar en Sostenibilidad (ERISOST). \\ Departament Sociologia i Antropologia Social, Universitat de València.
}

DOI: 10.1387/lan-harremanak.15439

\section{ABSTRACT}

La crisis ecológica continúa acumulando indicadores cada vez más alarmantes. Su resolución reclama un nuevo modelo de desarrollo humano, fundamentado en los principios de equidad y limitación del crecimiento a la capacidad de sustentación del planeta, que implica la necesidad de profundas modificaciones en los procesos de producción y en las relaciones sociales de producción. Requiere pues, resolver y decidir - de forma consciente- sobre el dilema que plantea la sostenibilidad para el futuro de nuestras sociedades tanto a nivel local como global.

En este trabajo se formula un marco teórico del campo de interacciones entre la "cuestión ecológica" y los sistemas de relaciones laborales, a partir del cual se desarrolla una propuesta de contenidos curriculares mediante núcleos temáticos y su articulación docente, atendiendo al perfil profesional de los futuros Graduados en Relaciones Laborales y Recursos Humanos.

Palabras clave: Dilema sostenibilidad, curriculum graduados en Relaciones Laborales, propuesta de contenidos y acciones para incluir la "cuestión ecológica». 
The ecological crisis continues accumulating increasingly alarming indicators. Its resolution claims for a new human development model, based on the principles of equity and maintaining growth limitation under the planet's sustainable capacity, which involves the need of deep modifications in the processes of production and its social relations. Therefore, it requires solving and deciding —consciously - about the dilemma sustainability poses for the future of our societies in both, local and global levels.

In this work is formulated a theoretical frame of the interactional field between the "ecological question" and the industrial relations system, from which a proposal for curriculum content through thematic cores and its educational articulation is developed, according to the professional profile of the future Graduates in Industrial Relations and Human Resources.

Key Words: Sustainability dilemma, curriculum graduates in Industrial Relations, content and action's proposal for "ecological question" inclusion. 


\section{Algunas consideraciones introductorias sobre la crisis ecológica: Insertar una aleta a un pájaro que necesita un ala mejor}

«Por tanto, la producción capitalista solo sabe desarrollar la técnica y la combinación del proceso social de producción, socavando al mismo tiempo las dos fuentes originales de toda riqueza: la tierra y el hombre.»

(Marx, K. 1982: 424)

«La propiedad asumida por el sistema como principio constitutivo es la propiedad que permite la máxima generalización de los procesos de individualización y, al mismo tiempo, es el principio que, incluido en el funcionamiento del sistema, termina negando toda determinación del sujeto que no sea puramente cuantitativa.»

(Barcellona, P. 1995:118)

«Si los conjuntos de individuos ya no forman pueblos sino sociedades de consumo, y el consumo está vedado al ochenta por ciento de la humanidad, el orden mundial depende de la aplicación de la tecnología de la represión y del olvido.»

(Galeano, E. 1996:41)

Crisis económica, social y ecológica son tres caras de una misma crisis. Son interdependientes y se retroalimentan entre ellas puesto que nuestro modelo de organización social y económica depende de los recursos naturales disponibles y, a su vez, la salud de nuestros ecosistemas (y por tanto de nuestro futuro) dependen de este modelo socio-económico (Guattari, 1990). La crisis económica capitalista no es una novedad en sí misma, en su continuado ciclo de expansión-contracción, aunque sí en su magnitud. Lo que resulta verdaderamente novedoso desde hace unas décadas es la experimentación de una crisis ecológica que llegó para quedarse y que, año tras año, se profundiza. Sin embargo, sus causas no suelen ser atribuidas al funcionamiento del sistema capitalista.

Por un lado, la globalización y las economías llamadas modernas están totalmente basadas en la energía y materias primas baratas, abundantes y de buena calidad. Por ejemplo, el transporte o el sistema agroalimentario dependen de los combustibles fósiles en general y del petróleo en particular. Durante la primera mitad de 2008, la crisis ecológica se tradujo en la subida exponencial de los pre- 
cios del petróleo y de los alimentos. La cotización internacional del barril del petróleo traspasó los 100 dólares y alcanzó un máximo histórico de 147 dólares en el mes de julio, en tanto que la denominada crisis alimentaria agravó la situación mundial del hambre. La Organización de las Naciones Unidas para la Agricultura y la Alimentación (FAO) señala que: en el primer semestre de 2008 los precios internacionales en dólares de los cereales habían alcanzado sus niveles más altos en casi 30 años [...]. Los precios de los alimentos eran un $40 \%$ superiores a los valores de 2007 y un $76 \%$ respecto a los de 2006 [...] la escalada de los precios de los alimentos empujó a unos 115 millones de personas al hambre crónica durante 2007 y 2008, lo cual significa que hoy en día viven en el mundo mil millones de personas hambrientas (FAO 2009: 6). Con todo, lejos de cuestionar la lógica mercantil subyacente, las recomendaciones que brindó la Conferencia de Alto Nivel sobre la Seguridad Alimentaria Mundial organizada por la FAO en Roma apuntaron al incremento de la productividad y de la producción. De modo que se instó a la comunidad internacional a que «intensifique la inversión en ciencia y tecnología para la alimentación y la agricultura» y "a continuar sus esfuerzos por liberalizar el comercio agrícola internacional, reduciendo las barreras comerciales y las políticas que distorsionan los mercados» (FAO 2008: 3). Mayor aplicación científica y tecnológica sobre la naturaleza y más mercado: las recetas propuestas no diferencian de las causas de la enfermedad.

Por otro lado, los impactos sobre el medio ambiente del sistema económico son hoy patentes. El cambio climático, de origen humano, es una amenaza para las generaciones futuras y nuestra economía: en caso de seguir los escenarios de Business as usual, los costes del cambio climático podrían ser superiores al 20\% del PIB europeo en los años venideros. Según la organización Global Humanitarian Forum para el año 2030 se espera que la vida de 660 millones de personas esté gravemente afectada, ya sea por desastres naturales causados por el cambio climático o por la degradación progresiva del medio ambiente (Global Humanitarian Forum 2009: 12). A pesar de la creciente preocupación de las potencias mundiales por el cambio climático, no han hecho más que crear los derechos de emisión de $\mathrm{CO}_{2}$ a partir del Protocolo de Kyoto, generando una suerte de privatización de la atmósfera. En la actualidad, el comercio de los créditos de carbono ascendió a 126.000 millones de dólares en 2008, y se espera que llegue a los 3,1 billones en 2020 (Friends of the Earth 2009: 4).

También la crisis ecológica se evidencia en el acelerado consumo de los bienes que provee la naturaleza. El informe Planeta Vivo de 2008 de la World Wide Fund For Nature (WWF) indica que en los últimos 35 años se ha perdido casi un tercio de la vida silvestre de nuestro planeta. Aún más impactante resulta el índice de huella ecológica, elaborado por la WWF, que mide la demanda de la población mundial sobre los recursos biológicos del planeta. La demanda de la humanidad en 1961 era la mitad de la biocapacidad mundial, mientras que en 2005 la demanda excedía en casi un $30 \%$ esa capacidad. Es decir que la huella ecológica aumentó 
más del doble en las últimas cuatro décadas y los pronósticos son menos alentadores, ya que a mediados de la década de 2030 la demanda equivaldrá a la capacidad biológica de dos planetas Tierra (WWF 2008: 2). La WWF atribuye los datos al crecimiento de la población mundial y al de los niveles de consumo, pero solo explica una parte del problema y adopta una posición cercana al neomalthusianismo.

La problemática ecológica envuelve aspectos económicos, sociales, culturales y políticos, de manera que requiere una visión global. Hoy, más que nunca, quedan expuestos los fundamentos del funcionamiento del modo de producción y reproducción capitalista como factores desencadenantes tanto de la crisis económica como de la crisis ecológica.

Así pues, la crisis ecológica habla. Habla de nuestro modelo de desarrollo civilizatorio, del tipo de crecimiento económico, de las características del sistema de producción, de los hábitos de consumo, de la disociación entre los procesos cíclico naturales y los procesos lineales humanos de transformación de la materia y energía y también habla de las políticas socio-económicas puestas en práctica que las atienden. Sus indicadores, en contrastada medida, son una muestra, un epítome circunstancial más de la insostenibilidad del crecimiento. Un modelo que no incluye las externalizaciones ambientales negativas (Martínez Alier y Schlüpmann, 1992; Naredo, 1993; Aguilera y Alcántara, 1994; García, 1995a) y que tiene su correlato en la no inclusión, o como mucho en una consideración sesgada, de los costes reales en los balances económicos. Junto a ello, un modelo donde se produce una dilución de atribuciones y responsabilidades propia de las formas cada vez más complejas de organización social, de su estructura y de su estructura de poder (Ovejero, 1.989), donde nadie parece ser responsable, obstaculizando una planificación de la curva sigmoidea (Hadeler, 1982) y donde , junto a su actual sistema de producción postfordista de matriz industrial (Gorz, 1988; Lipietz, 1997; Alonso, 1999) y altamente generador de entropía (Georgescu-Roegen, 1975), adquiere una centralidad determinante nuestro modelo de consumo (Alonso, 2006). Si bien ya de por sí la especie humana se caracteriza por un consumo exosomático (Martínez Alier, 1995; García, 1995b) para cubrir sus necesidades vitales, a ello, se une socialmente, por una parte unas pautas de consumo basadas en bienes posicionales (Hirsch, 1976; Alonso; 2005;2006) —a partir de los que se establecen culturalmente nuestros aspectos identitarios de status, poder, prestigio- $-\mathrm{y}$, por otra, las exigencias derivadas de un sistema de producción capitalista, donde la máxima de la rentabilidad económica es el pilar sobre el que se articula dicho modelo de consumo (Altvater 1994, Riechmann, 1998; Gorz 1995).

No basta con el análisis de la relación capital-trabajo, sino que se vuelve imprescindible incorporar una nueva mirada de la relación entre el hombre y la naturaleza y, específicamente, el modo en que el régimen capitalista de producción se apropia de su entorno natural. La producción capitalista en escala ampliada se apoya en un mundo natural crecientemente mercantilizado, que no solo provee de valores de uso, sino también que adquiere un precio mediante el cual puede ser 
enajenado y apropiado (Altvater, 2003; Sabbatella, 2009). En la subsunción real, la naturaleza se presenta como una fuerza productiva del capital, «es, cosificada, desnaturalizada de su complejidad ecológica y convertida en materia prima de un proceso económico; los recursos naturales se vuelven simples objetos para la explotación del capital» (Leff 2005: 264). Esta apropiación será entendida en términos de la subsunción real de la naturaleza al capital, de una «racionalidad que separa en un primer momento recursos naturales de otros componentes no valiosos de la naturaleza, incapaces de servir como fuentes de valorización capitalista; y en un siguiente paso, esa racionalidad separa un recurso natural del otro» (Altvater 2009: 3).

El régimen capitalista no solo incluye a la naturaleza, sino que también la subordina a los designios de la producción de plusvalor, generando la conocida como segunda contradicción del capitalismo (O'Connor, 1990; 2001;). Es un proceso simultáneamente extensivo e intensivo. Extensivo porque el capital se va adueñando de cada porción de la naturaleza, ampliando las fronteras de extracción como continuidad de la acumulación originaria o primera contradicción del capitalismo. E intensivo porque cada vez precisa mayor cantidad de bienes naturales y de sometimiento de las fuerzas naturales y del factor trabajo para incorporarlos como medios de vida y medios de producción, fundamentalmente como energía y generador de una aceleración entrópica en su ya conocida dinámica de la lógica (ilógica) de la rueda de la producción (Schnaiberg, 1980; Gould y Schnaiberg, 1996):

La lógica de la «rueda de la producción»

1. Incrementar la acumulación de riqueza, mediante la propiedad de organizaciones económicas que usan los recursos ecológicos para expandir la producción y los beneficios (capital versus naturaleza).

2. Incrementar la tasa de asalariados (empleados y autónomos), a fin de generar dependencia de éstos respecto de la expansión de la producción para poder mejorar en cuanto a puestos de trabajo y salarios (capital versus trabajo).

3. Incrementar la asignación de riqueza acumulada a nuevas tecnologías a fin de sustituir trabajo por capital físico y generar así más beneficios para los propietarios de la riqueza, de modo que éstos puedan sostener y aumentar su propiedad frente a la competencia creciente de otros propietarios de riqueza (capital versus capital).

4. Incrementar las actividades de los gobiernos dirigidas a facilitar la acumulación ampliada de riqueza, de acuerdo con objetivos de «desarrollo nacional», por una parte, y de «seguridad social», por otra (capital versus gobernanza).

5. El resultado neto de los anteriores procesos es una necesidad creciente de extracciones (de recursos) y adiciones (de residuos) ecológicas a fin de sostener un nivel determinado de «bienestar social».

6. El anverso ecológico del punto 5 es el incremento continuo de desorganización ecológica, a medida que las presiones económicas empujan hacia una mayor extracción de valores mercantiles a partir de los ecosistemas.

7. Como extensión del punto 6 , las sociedades se tornan crecientemente vulnerables a la desorganización socioeconómica, a medida que su base de recursos ecológica resulta ella misma desorganizada. 
Más allá de cómo el capital supere su crisis económica, no puede superar por sí mismo la crisis ecológica a la cual ha sometido al mundo entero, por más que frente a la segunda ley de la termodinámica se esté articulando un complejo científico-técnico basado en las nuevas formas de biotecnología y/o nanotecnologías capaces de, a partir de la propia naturaleza, crear una naturaleza paralela artificial patentada, sobre un modelo que haga compatible la ilimitada acumulación capitalista y los límites de la naturaleza, entre la reproducibilidad y circularidad del capital, como bien ejemplifica el obtuso e imposible (empíricamente) concepto de desarrollo sostenible. «Las crisis económicas son cíclicas. La crisis ecológica no tiene retorno; por el contrario, se profundiza en tanto se mantengan vigentes los fundamentos de la presente formación histórica, económica, política, social y ambiental»(Sabbatella, 2009:21). Posiblemente el problema ambiental no sea más que una manifestación de una crisis global que afecta a los mismos fundamentos del modelo actualmente dominante de sociedad, característico del capitalismo avanzado (Daly; Schütze; Beck; Dahl, 1996; Goodland; Daly; El Serafy;Von Droste; 1997), y donde la cuestión ambiental como problema ecológico y de los recursos naturales a medio plazo, está más en factores socioeconómicos y políticos inherentes a los procesos de sobreexplotación, formas de apropiación y distribución, relaciones económicas internacionales, soberanía sobre los recursos, nueva división internacional del trabajo y relocalización industrial etc.. que en los límites físicos del planeta para sustentar un desarrollo de la humanidad, por imponderables a largo plazo que éstos sean y nos obliguen a un imperativo de decrecimiento (Jiménez, 2001; Folch, 2011; Riechman, 2006).

Es pues difícil de imaginar un desarrollo sostenible, que en primera instancia continúa negando un acceso equitativo a los recursos, y donde lo ético ha sido desplazado por una estética del simulacro de base tecnológica. El dilema se agrava si como apuntan tanto Norgaard (2009) como Sachs (1999; 2007) la sostenibilidad es además una cuestión de equidad (distribución de los derechos) entre generaciones, y no una cuestión del uso eficiente de los recursos y de los sistemas medioambientales, ya que considerando que las generaciones futuras, en el proceso de realizar los cambios que conducen a una mayor eficiencia, adquieren derechos adicionales a los recursos, una economía insostenible continuará siendo insostenible, aunque de manera más eficiente. El actual modelo de globalización, basado en un imperialismo exosomático, no hace sino agravar aún más el dilema, mediante la destrucción continua y sistemática no sólo de los recursos naturales sino también mediante el desmantelamiento de las formas de autonomía colectiva (Riechmann, 2006) Como gráficamente señaló GeorgescuRoegen (1994) el modelo se expande insertando una aleta a un pájaro que necesita un ala mejor. 


\section{Medio ambiente, relaciones laborales y recursos humanos: elementos para la configuración de un marco conceptual de referencia y de acción para el diseño de objetivos y núcleos temáticos}

En este apartado, se trata de dar cuenta de aquellos elementos que permitan delimitar el ámbito de estudio resultante de la interacción del nuevo contexto ecológico con los sistemas de relaciones laborales, considerados en su concepción amplia y abierta, entendiendo que ello sólo puede ser abordado desde una pluralidad multiparadigmática y, que permita atender ese objeto de conocimiento en su multiplicidad, variedad, complejidad, procesualidad, sin constreńirlo y por lo tanto ubicándolo necesariamente en su multidisciplinariedad. Como hemos reseñado ya con anterioridad, la crisis ecológica contemporánea supone una crisis del modelo de desarrollo civilizatorio, de la civilización industrial. El modo de producción, está pues en el centro del problema y si éste tiene algún viso de solución, pasa necesariamente por una profunda reconversión de los procesos productivos y de transformación de las relaciones sociales de producción (Hildebrandt, E., 1992).

Surgen así diversas cuestiones ante este doble imponderable ¡«Esa reconversión ha de ser sólo producto de las direcciones empresariales, de la actividad política, científico-técnica, de la presión de los movimientos ecologistas?», ¿los trabajadores y trabajadoras serán sólo un objeto pasivo de los cambios?, ¿las experiencias y saber-hacer acumulados en el mundo del trabajo no son más que un elemento marginal en el proceso? ¿la reconversión de las empresas afecta sólo a los trabajadores trabajadoras en su condición de ciudadanos y no como agentes productivos, tanto a nivel individual como colectivo?. ¿la gestión de la mano de obra no requiere de la implicación y de la participación de los trabajadores? ¿Acaso las nuevas medidas reguladoras del medio ambiente no imponen restricciones a la actividad empresarial y con ello a las relaciones laborales?

Un largo etcétera de interrogantes, evidencia la emergencia de que no puede concebirse un cambio de las tecnologías, de su aplicación, de los productos o las formas de acceso a los mercados y consumo, sin que ello implique también un cambio en los agentes de las relaciones laborales y en sus formas de interacción (Lerma, I. y García, E., 1997). El ámbito de la producción (primaria, industrial o de servicios) es un universo de relaciones sociales, que como tal no queda al margen de las nuevas demandas.

Como ha señalado García Ferrando, (1994), «el cambio de perspectiva de análisis de los problemas ecológicos, desplazándose desde la óptica del crecimiento cero a la del desarrollo sostenible, que, para algunos analistas representa una pérdida de impulso, impone, en realidad, una carga más pesada a la em- 
presa, los principales agentes económicos y los distintos actores sociales. Los modos de producir, diseñar tecnología, consumir, necesitan ser alterados, mediante ensayo y error, en un esfuerzo cooperativo de distribución de cargas, ajustando mutuamente contribuciones y demandas. El objetivo de garantizar estándares de vida elevados y conservación del entorno, cristalizado en el programa de construcción de un ecosistema industrial de nueva planta, representa seguramente unos de los retos más formidables y, a la vez, imperiosos a los que ha hecho frente la especie humana hasta hoy" (1994:26-7).

La crisis ecológica, demanda pues una respuesta de la sociedad y dicha respuesta involucra a todos sus ámbitos. Los sistemas tradicionales de relaciones laborales, aun estando en la actualidad acusando una aguda transformación en cuanto adaptación a las exigencias del nuevo ciclo económico (mayor o menor presencia sería un debate que en definitiva nos remitiría a atender sus modificaciones cualitativas internas) son también performados por el impacto de dicha crisis. Atender sus modificaciones, las modificaciones en los actores, en sus prácticas y en sus estrategias (conflictivas o consensuadas) ante dicho imperativo constituye el marco de referencia analítico que pretendemos establecer (Lerma, I. y García, E., 1997a).

Sobre la base de lo anteriormente expuesto, cabe pues distinguir diferentes niveles que pueden ser explicitados atendiendo a los componentes que involucran.

Un primer ámbito de ampliación de las relaciones laborales a la cuestión ecológica, puede ser considerado a partir de elementos clásicos de las relaciones laborales. En un principio, el punto de partida puede encontrar su anclaje a través de dos elementos consustanciales de las relaciones laborales que se ven afectados por el imperativo ecológico. Aunque ambos son elementos clásicos de las relaciones laborales, no obstante, no son elementos estáticos sino que por el contrario están en continua modificación y adaptación:

a) Conservación, creación y modificación de empleos y protección del medio ambiente.

b) Protección de la salud y seguridad y protección del medio ambiente.

El primero de ellos, no sólo tiene un carácter relevante de centralidad en las relaciones laborales, sino que además cobra mucha mayor vigencia en la actualidad, ya que empleo y medio ambiente es una pareja de moda popularizada por sus respectivas crisis. Con todo, siendo indeterminada la cuantificación de los posibles empleos generados por un sector medioambiental — que está sin delimitar de ahí la escasa fiabilidad de las proyecciones - un imperativo de reconversión de procesos recorre el sistema productivo (Hildebrandt, E., y Oates, E., 1997; Renner, M. 1994; Riechman, J., 1998). 
El segundo remite a la redefinición y extensión del concepto de salud, como ampliación hacia la salud integral del individuo y de las comunidades, sobre la base de las actuales formulaciones y tendencias de esta nueva concepción emanadas de las instituciones de política de salud pública (Rodríguez, M., 1995; Dolz, J., 1990).

Por otra parte, también como punto de partida o de entrada, pero desde el interés empresarial, se puede considerar a su vez dos ámbitos sobre cuyo desarrollo se implica una gestión medioambiental para las empresas:

a) Extensión de la cuota de mercado.

b) Imagen de la empresa.

En este caso, el primero de ellos remite tanto a un cierto volumen de negocio emergente con relación a las estrategias de absorción de una cuota de ese volumen, como por otra parte a las cuotas que se pueden adquirir por innovación y los beneficios derivados de ésta. En ambos casos, su desarrollo es variable en función de la demanda de mercado, pero también ésta se ve afectada por la capacidad de las empresas de generar dicha nueva demanda (Duclós, D., 1991; Hildebrandt, E., y Schmidt, E., 1994).)

Junto y asociado a ello, los beneficios derivados de una imagen corporativa adecuada y lo que se denomina compromiso social de la empresa, conforman su consecución en un entorno altamente competitivo.

Así pues, la combinación de los dos elementos clásicos, que hemos reseñado más arriba, con proyección ecológica en un nuevo entorno competitivo del que las empresas es difícil que se sustraigan, permite delimitar un primer escenario donde la dinámica de las relaciones laborales, del juego de intereses, polariza a priori - pero sin patrimonializar izar en exclusiva - los diferentes elementos sobre los actores. Desde ambos juegos de intereses, y ante la presión del entorno que obliga a las partes, es posible entonces delimitar una zona de consenso, aunque mínima, a partir de la cual las relaciones laborales y sus actores pueden ensanchar su contenido en cuanto se ven afectadas por la variable ecológica (ver cuadro 1): 


\section{Cuadro 1}

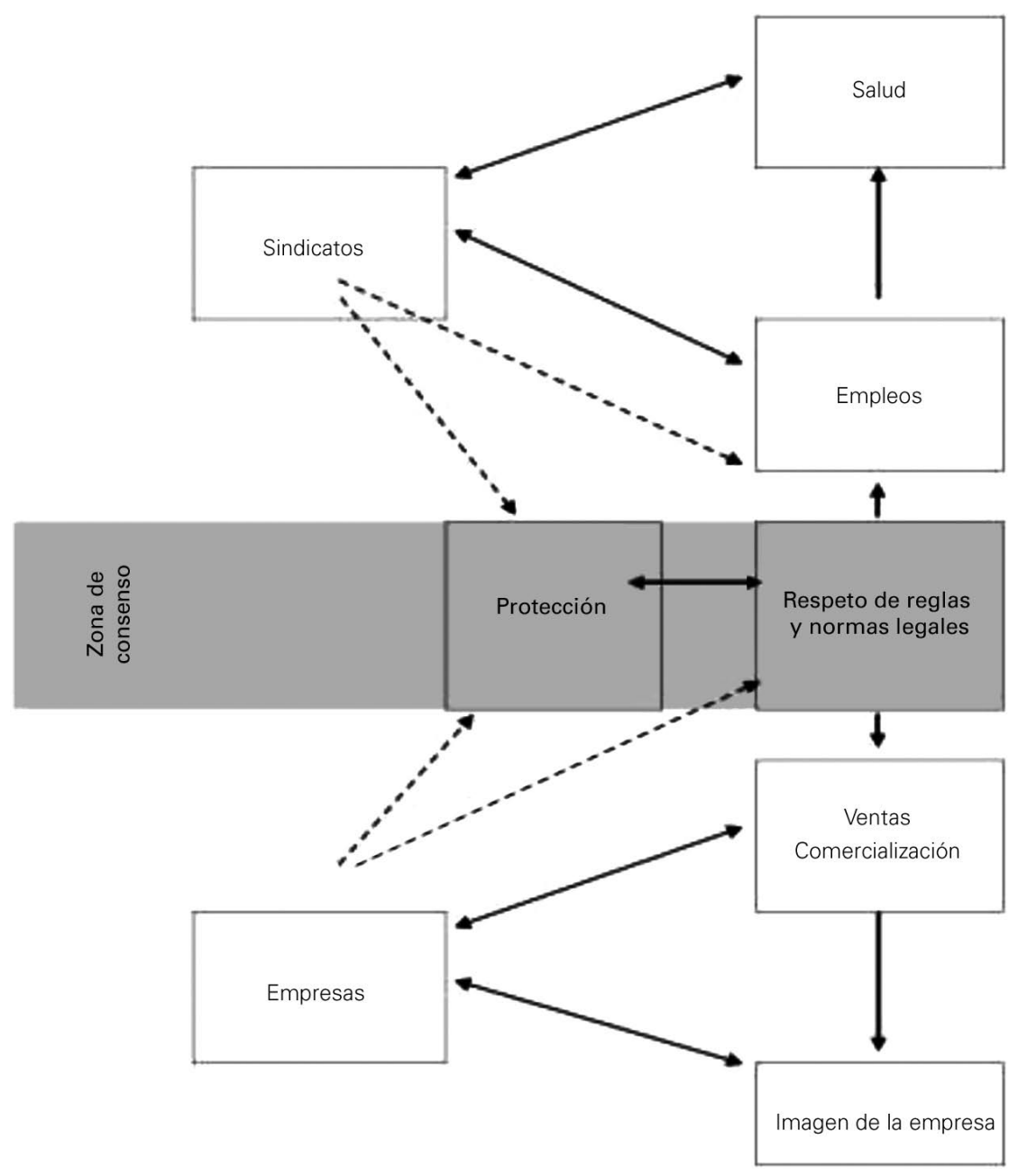

Ahora bien, salud y puestos de trabajo no agotan ni pueden, a través de las estrategias que sobre ambos se apliquen, dar satisfacción a toda la temática derivada de la cuestión ecológica. De hecho, aunque respecto al primer asunto se puedan establecer aumentos de competencias a través de los denominados comités de salud laboral y en el caso de la reconversión de puestos de trabajo se pueda mediar a través de los comités de empresa, en ambos casos sus competencias están o normativamente restringidas en el primer caso o son dependientes muchas veces de criterios que se establecen en ámbitos superiores y con variables que escapan a la voluntad de las partes. 
Con todo, algunos de estos contenidos se mueven en las rutinas negociadoras y/o están también fuertemente mediados por conformaciones culturales como, por ejemplo, la medicalización de la salud. Junto a ello, no puede obviarse que las empresas tienden a restringir la toma de decisiones estratégicas tanto internas como externas, pues es potestad normativa de sus propietarios, y con ello estrechan el objeto de las negociaciones a las condiciones y a la organización del trabajo en sus aspectos más clásicos e instrumentales (a excepción de las innovadoras). Así mismo, trabajadores y sindicatos intercambian penosidad y peligrosidad por una batería significativa de pluses y compensaciones económicas. Como se suele decir vulgarmente, el tema de las condiciones de trabajo es de los primeros que se cae de la mesa de negociación.

Sin embargo, si lo anteriormente señalado puede modificar, restringir o velar unas prácticas internas de funcionamiento, no por ello elimina el problema, sino que explicita una dimensión de éste, que debe ser reconocida y comprendida en sus múltiples dimensiones que la conforman. Sin ánimo de exhaustividad, son elementos determinantes: el tamańo de la empresa, su ubicación en las redes productivas y de distribución, el papel de las instituciones públicas con competencias en la materia, la cultura o culturas de la empresa, y toda aquella escala de puntos intermedios que se pueden establecer en un arco que recorre desde los derechos de los propietarios hasta los mecanismos de participación (Lerma, I. y García, E., 1997b).

Así pues, en este nivel de extensión pese a sus potencialidades se puede señalar sus carencias, esto es, la no consideración de la totalidad de los problemas a los que se unen ciertas prácticas que diluyen la propia emergencia del problema ambiental.

Un segundo ámbito de extensión de las relaciones laborales hacia la atención de la problemática ecológica, más allá de sus contenidos clásicos, permite ahondar en la cuestión, al mismo tiempo que denota un nivel creciente de complejidad.

Este segundo ámbito se puede puntuar desde la emergencia de un nuevo campo vinculado a la necesidad social de constituir una política medioambiental, y que dadas sus características como potencial elemento regulador o conformador de otras políticas, no escapa a la atención de los actores sociales de las relaciones laborales, pues necesariamente los implica (Oates, A., y Gergory, D., 1993). Ello requiere atender a su vez la dinámica más amplia que dichos actores desarrollan a través de los diferentes niveles de negociación. Estos pueden ser considerados como escenarios o distintas instancias donde conflicto y consenso cobran carta de naturaleza, dependiendo de las circunstancias, (coyuntura y estrategia de los actores) pueden variar en cuanto a su extensión, influencia y capacidad de regulación y de articulación entre ellos. Se deben considerar así: 


\footnotetext{
LA INCLUSIÓN DE LA "CUESTIÓN ECOLÓGICA» EN EL CURRÍCULO

De los/as graduados/as en Relaciones Laborales y Recursos Humanos
}

— el nivel superior de ámbito nacional (macro)

- el nivel intermedio sea de ámbito sectorial o territorial (meso)

- el nivel inferior de la empresa sobre la cual interactúan directamente las direcciones de las empresas con los diferentes grupos de interés o, trabajadores individualizados (micro).

Junto a ello, habría que implementarle un cuarto nivel superior, derivado del proceso de integración europea, cada vez más relevante, en tanto que la comunidad de mercado impone determinadas orientaciones y la satisfacción de éstas para competir en él, a lo que se une la capacidad legislativa de las instancias superiores europeas sobre las que en buena medida, los Estados descargan responsabilidades (sobre todo en legislación y política medioambiental, tanto sobre productos, como niveles admitidos, como procedimientos, configurando una regulación administrativa normativa u orientadora según los casos).

La consideración de estos niveles remite pues a la existencia de un nuevo ámbito, esto es, el de la política ambiental que dadas sus repercusiones ampliaría los contenidos tradicionales de las relaciones laborales, y que además dadas sus características de transversalidad, mediaría horizontalmente entre las políticas de base que afectan tradicionalmente al sistema de relaciones laborales, cuya resultante son determinadas políticas laborales y los diferentes ámbitos de negociación donde interactúan los actores sociales y donde sus organizaciones y asociaciones desarrollan diferentes estrategias (García, E., y Lerma, I., 1994). Expresado gráficamente, (ver cuadro 2 ):

El cuadro 2, expresa pues una multiplicidad de campos o escenarios y una complejidad que deben ser atendidos en sus propias especificidades.

El escenario superior-macro, junto a los contenidos que se puedan desarrollar a través de él, también es un campo privilegiado tanto para la descarga de responsabilidades como para la exigencia de medidas que articulen la política medioambiental, su financiación, secuenciación, adaptabilidad, flexibilidad y todos aquellos otros elementos que posibiliten un timing adecuado a la reconversión ecológica del sistema productivo. Junto a ello, también es el campo de interacción que permite transmitir las percepciones y los avances que se producen y delimitar o constreñir las actuaciones de las otras partes.

Los dos otros campos de negociación, en cuanto escenarios más de aplicación adquieren también su relevancia.

En el contexto por ejemplo de nuestro país, dada su articulación territorial en base a Comunidades Autónomas, manifiesta la presencia de mesogobiernos con capacidad interventora también sobre el binomio medio ambiente-relaciones laborales, y donde por lo tanto a su vez se reproducen ciertas características del nivel macro. Ello a su vez complejiza la política medioambiental, ya que las disimetrías que se pueden establecer otorgan diferentes capacidades o situacio- 


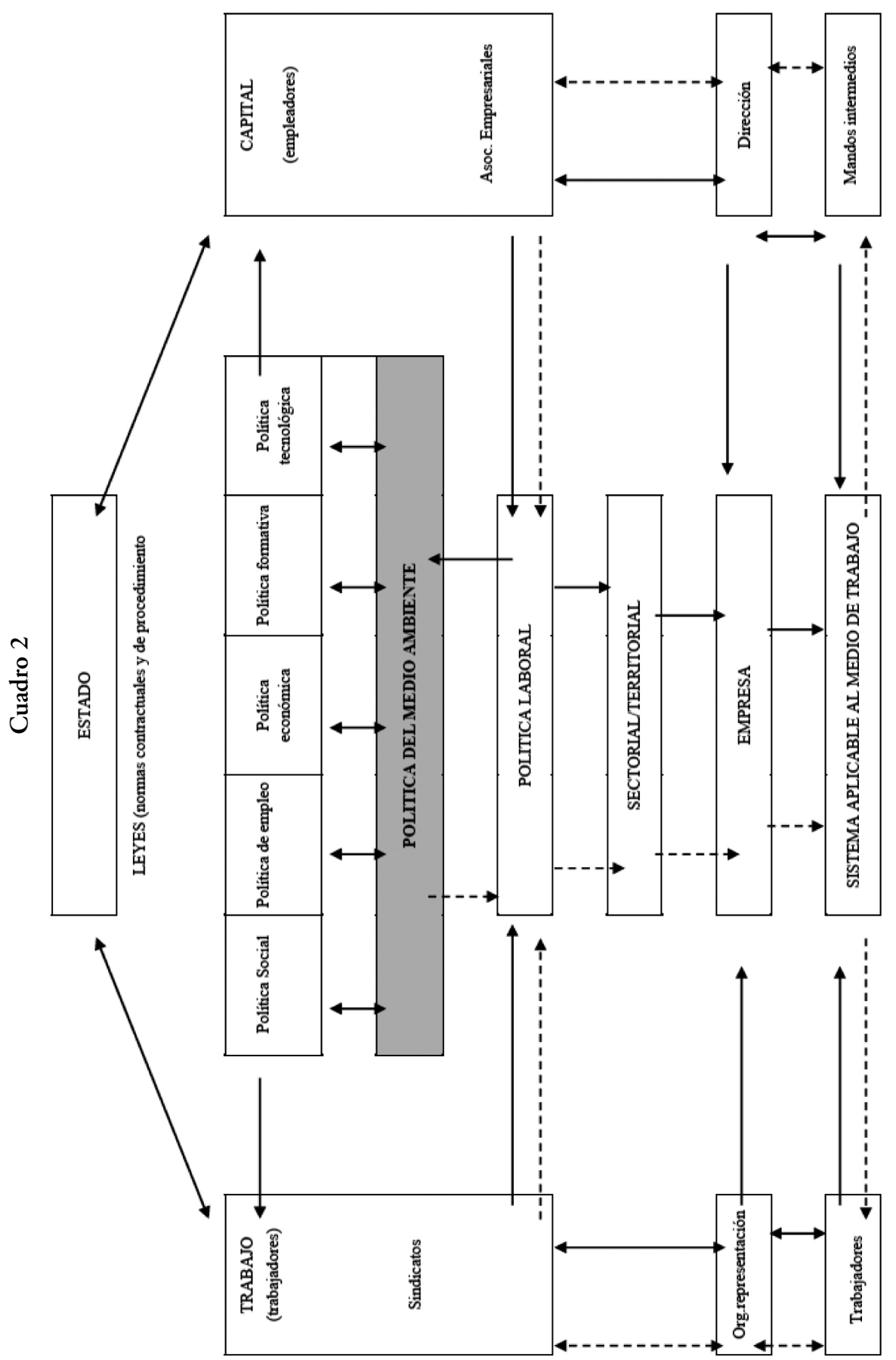


nes de dumping ecológico (La Roca, F. y Sánchez, A., 1996). Por otra parte, lo anteriormente señalado no elimina la centralidad de la empresa como campo de actuación. No en vano, en ella es donde en primer lugar se generan potencialmente los riesgos y peligros ecológicos; en segundo lugar la empresa tiene una cierta titularidad en las estrategias que permitan minimizar esos riesgos y, en tercer lugar sobre ellas recae el problema de la polución, la no utilización de productos contaminantes y peligrosos.

Como ha señalado García Ferrando (1994), «las empresas están sometidas de manera creciente al fenómeno de «sobrecarga de demandas», procedentes de sectores muy diversos - frecuentemente con intereses, visiones y exigencias diferenciadas, cuando no contrapuestas-, y quien está al frente de la dirección de aquellas se ve obligado a gestionarlas e integrarlas en un paquete coherente y viable. Demandas tales como el mantenimiento y creación de puestos de trabajo, la mejora de la competitividad, el incremento de la calidad de bienes y servicios acompañada de reducción en los precios, el respeto al medio ambiente natural y un largo y permanentemente abierto listado de exigencias, que obligan a las empresas a dotarse de extraordinarias dosis de flexibilidad, innovación y capacidad de gestión del riesgo, sin perder el sentido de lo que resulta, en cada momento, empíricamente viable o compatible con la sobrevivencia misma de la empresa» (1994:14).

Recapitulando, lo expresado hasta ahora remite al escenario o escenarios de intervención. Dichos escenarios, no niegan sino que por el contrario evidencian que la empresa es un espacio de relaciones sociales, pero que éstas están inscritas también en un universo de relaciones sociales que permeabilizan las paredes de las unidades productivas y de las relaciones laborales a las influencias del entorno o contexto en el cual deben de operar. Nuevamente los imperativos de mercado deben ser considerados pues cualquier optimización que se pretenda articular, no lo puede ser en el abstracto, sino en un nuevo contexto concreto operacional.

Ello, abre el binomio relaciones laborales-medio ambiente, a los cambios que se producen en el entorno y para los cuales los puntos de partida iniciales a través de sus contenidos clásicos quedan estrechos como hemos señalado. Dicha apertura de las relaciones laborales clásicas, puede ser considerada como reactiva, reactiva-estratégica o por iniciativa-estratégica, en cuanto a la consideración de los problemas ecológicos, pero implica necesariamente la integración de nuevos factores y de nuevos procedimientos para su instrumentación, junto a los existentes así como de las modelizaciones y variaciones futuras que de ambos se puedan producir (Lerma, I., y García, E., 1997b). Se pueden delimitar así tres nuevos elementos/ámbitos de extensión del sistema de relaciones laborales en la medida de su aceptación de la variable ecológica:

a) Extensión de los temas y contenidos.

b) Extensión de los actores.

c) Extensión de las formas de regulación. 
El primer elemento/ámbito puede a su vez ser desagregado en función de los diferentes niveles de negociación. En este sentido, se pueden constatar a nivel macro, contenidos como la reforma fiscal ecológica, programas de financiación para la reconversión ecológica de las empresas, la inversión pública en actividades productivas potencialmente generadoras de puestos de trabajo vinculadas a resolución de problemas ambientales, la financiación diseño y ejecución de campañas y programas de formación o de reciclaje de los trabajadores. A nivel meso, se señalan acciones específicas de ámbito territorial que desarrollan programas de ámbito superior o bien programas específicos adaptados a las peculiaridades socioproductivas, así como por otra parte planes y programas sectoriales, cuya temática es variada, desde la optimización de recursos, la gestión de bolsas de subproductos, el tratamiento de los residuos etc... En el ámbito de la empresa o nivel micro, la minimización de residuos, la reorganización de tiempos y cargas de trabajo, las compensaciones económicas, la negociación de la formación, la implementación de nuevos productos etc... (García, E.-La Roca, F./Hildebrandt, E. y Külhleis, D. 1996). Todos ellos pueden encontrar su materialización bien en Acuerdos o Pactos, bien en la negociación colectiva materializada en los convenios (ver cuadro 3)

\section{Cuadro 3}

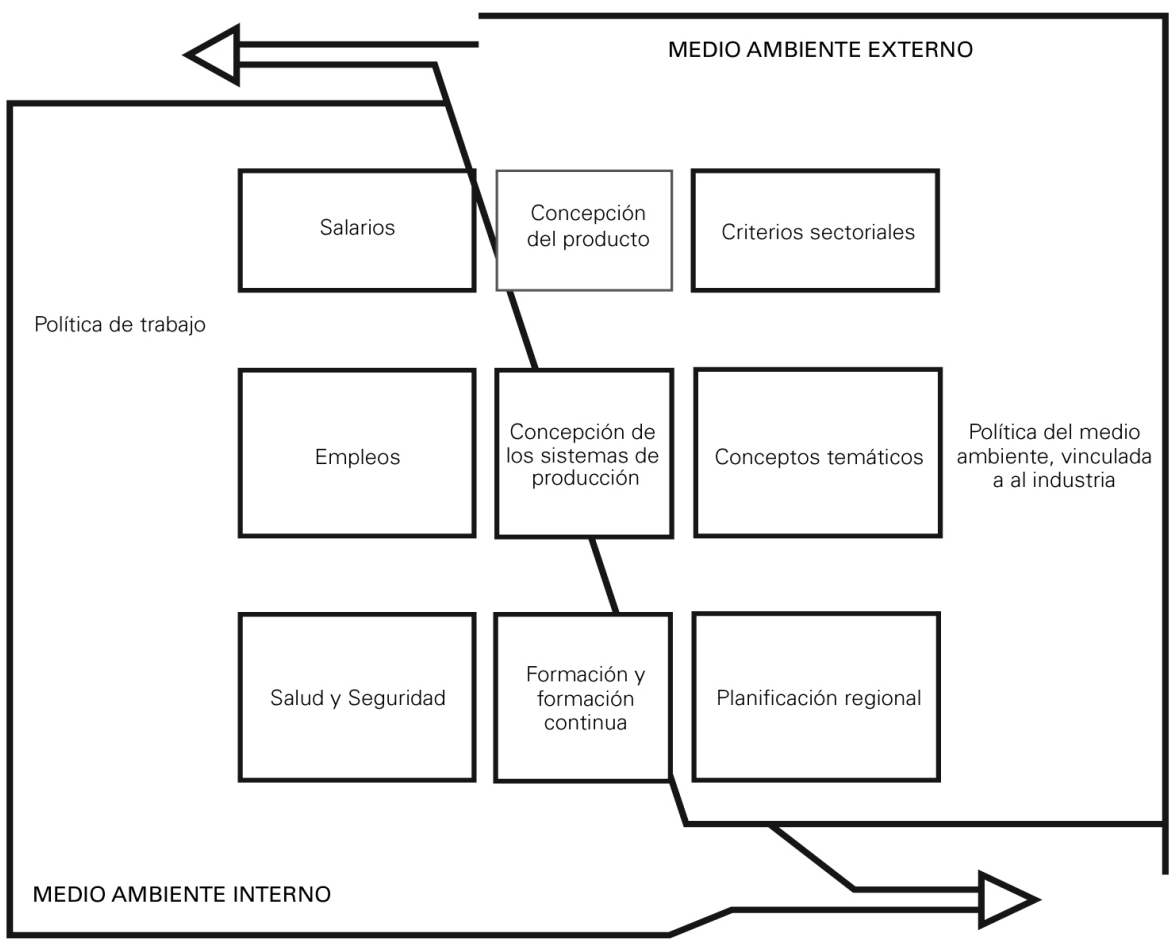


El segundo elemento hace patente la extensión del juego tripolar (empresarios y sus asociaciones, trabajadores y sus sindicatos y Estado y organismos con competencias en las relaciones de trabajo) a nuevos actores del entorno.

Ello es debido a que la problemática ecológica, no sólo evidencia la permeabilidad de las paredes del sistema productivo, sino que abre por sus características una zona intermedia de conflicto (nuevas demandas) que escapa a la posible totalidad de su control por parte de los sujetos negociadores clásicos.

Quizás uno de los elementos determinantes, de la apertura del sistema de relaciones laborales deba ser entendido desde la ampliación a otros sujetos (entidades locales, grupos ecologistas, respuesta a los consumidores) que median, construyen, delimitan e intervienen de una manera creciente en los pequeños o grandes conflictos ecológicos (macro, meso, micro) que se originan derivados de los procesos de producción. Junto a ello, de cara a la estrategia de los actores sociales, no puede ser minimizado el cambio cultural por incremento de sensibilidad ecológica en general de la población, así como la influencia de los media.

\section{Cuadro 4}

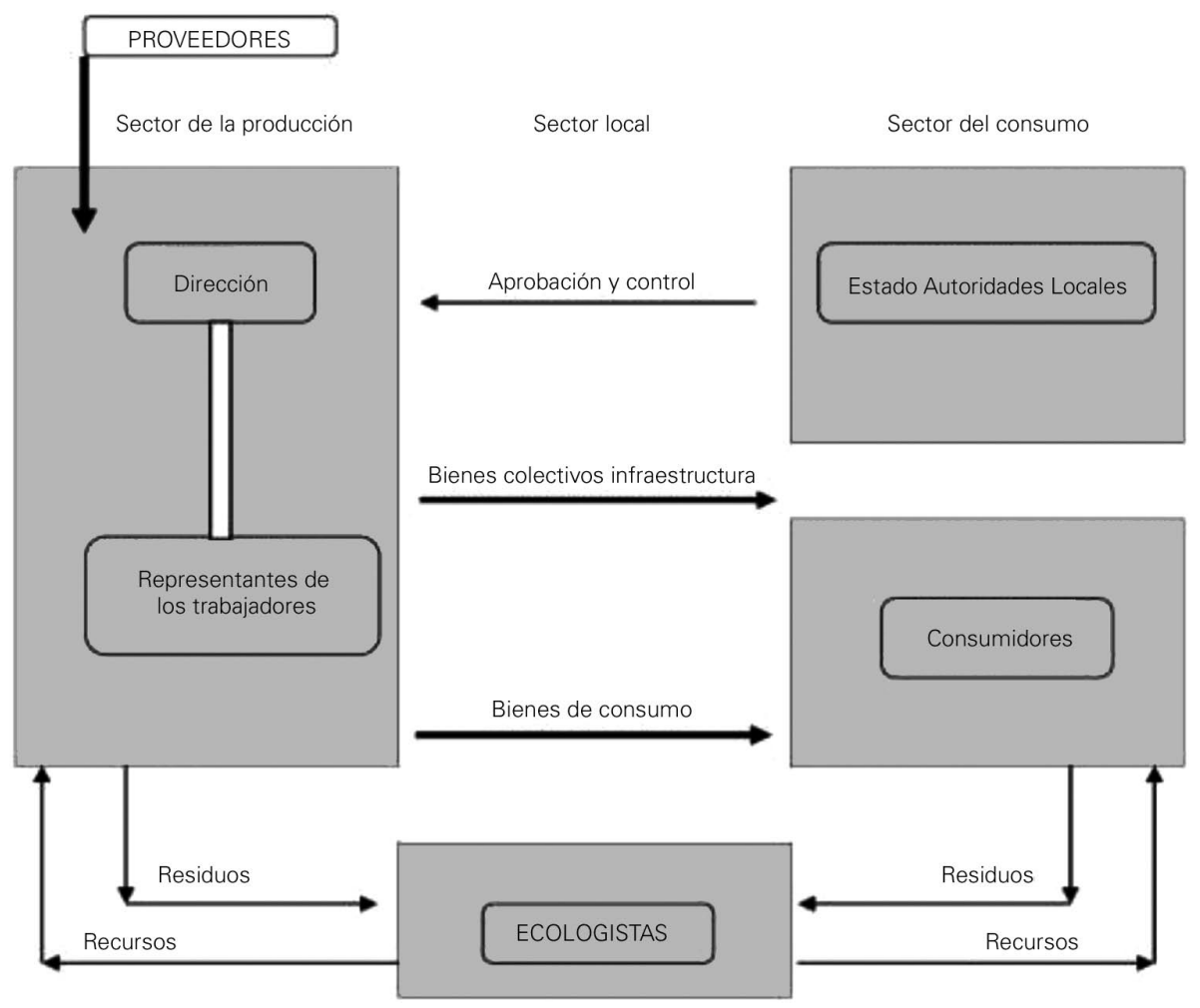


El circuito restringido de las relaciones laborales clásicas, amplía así el número de interlocutores, redefine las estrategias y prácticas de los actores sociales clásicos, en función de la capacidad, estrategias y prácticas de estos nuevos sujetos (cuadro 4).

El tercer elemento, la extensión de formas de regulación, esto es, referidas no a sus aspectos reglamentarios normativos, sino a las prácticas consuetudinarias, debe atender dos dimensiones.

En primer lugar, la extensión de las relaciones laborales a la hora de atender la problemática ecológica, y dar respuesta satisfactoria a las nuevas demandas y a los nuevos contenidos, difícilmente puede asentarse sobre las rutinas negociadoras, propias de los contenidos clásicos de las relaciones laborales (salarios, horarios, pluses, productividad...).

En la medida en que se constituye un campo intermedio que escapa a la totalidad del control de los actores clásicos y dada una indeterminación de escenario de conflicto que no exime en su afectación a las partes, dichos contenidos encuentran un mayor acomodo, en el ámbito de prácticas negociadoras blandas (soft bargaining) que ofrecen un espacio para diseñar estrategias flexibles y dúctiles.

Así pues, ello no significa que la inclusión de la problemática medioambiental origine nuevas prácticas de regulación, sino que más bien refuerza una tendencia ya asentada en mayor o menor grado, ampliándola por ello. Esta tendencia a la creación de un campo de consenso en el escenario negociador formal o informal, puede ser considerada desde diversas ópticas, bien como resultante de una asimetría mayor entre las partes, derivada de los procesos de segmentación del mercado de trabajo, bien derivada de acuerdos y estrategias corporativistas, bien derivada de que en un entorno cada vez más competitivo y cada vez más complejo resulta difícil integrar nuevos problemas verticalmente o, bien por la conjunción de todos ellos (Lerma, I., y García, E., 1997a).

En segundo lugar debe considerarse su inserción a través de fórmulas de participación directa, en un escenario de por sí autoconsensuado, delimitado cada vez más por las nuevas formas de organización del trabajo (círculos de calidad, grupos autónomos o semiautónomos de producción) o por cualquiera de las múltiples formas que éstas pueden adquirir dependiendo del tamaño de la empresa, pero que en definitiva articulan modelos graduales de regulación del consenso sobre base integrativa por objetivos negociados: 
LA INCLUSIÓN DE LA "CUESTIÓN ECOLÓGICA» EN EL CURRÍCULO

Cuadro 5

\begin{tabular}{|c|c|c|}
\hline Elementos & Prácticas clásicas & Prácticas consensuales \\
\hline Resultados & Beneficio-pérdida & Suma positiva \\
\hline Participación & Obligatoria & Voluntaria \\
\hline $\begin{array}{l}\text { Estilo de } \\
\text { interacción }\end{array}$ & Indirecta & Directa entre las partes \\
\hline Procedimientos & Mismas reglas para cada caso & $\begin{array}{l}\text { Nuevas reglas y procedimientos, } \\
\text { según los casos }\end{array}$ \\
\hline $\begin{array}{l}\text { Métodos } \\
\text { de cierre }\end{array}$ & Imposición de una decisión & $\begin{array}{l}\text { Aceptación voluntaria de una de- } \\
\text { cisión elaborada por las partes }\end{array}$ \\
\hline Coste & $\begin{array}{l}\text { Débil a moderado a corto plazo } \\
\text { y elevado a largo plazo }\end{array}$ & $\begin{array}{l}\text { Moderado a elevado a corto tér- } \\
\text { mino y débil a largo término }\end{array}$ \\
\hline Representación & $\begin{array}{l}\text { Carácter general, responsables } \\
\text { electos o nominados }\end{array}$ & $\begin{array}{l}\text { Ad hoc, selección específica para } \\
\text { cada caso }\end{array}$ \\
\hline
\end{tabular}

Se puede entonces delimitar un nuevo escenario que no sustituye necesariamente para todas las casuísticas, el derivado de la ampliación sobre la base de los contenidos clásicos de las relaciones laborales. Seguramente existirán situaciones de solapamiento, o de preponderancia de un modelo sobre otro, pero lo cierto es que en base a las nuevas demandas y al desarrollo de una política medioambiental trasladable al ámbito de las relaciones laborales, surgen nuevas figuras y nuevos procedimientos dirigidos a optimizar las partes sus estrategias en un entorno que aún siendo cada vez más competitivo, sigue manteniendo un nivel de exigencia en cuanto a consideración de la problemática ecológica (García, E.La Roca, F./Hildebrandt, E. y Külhleis, D., 1996).

Conviene no obstante señalar que dicho entorno, donde las relaciones laborales han sufrido un fuerte proceso de empresarialización y donde es más que detectable en materia medioambiental el paso de mecanismos de policía administrativa (regulación normativa de la conducta del particular y sanciones por incumplimiento), a mecanismos basados en regulaciones pactadas, es decir acuerdos entre las administraciones y las empresas, está dando lugar a la configuración de un nuevo modelo de regulación y de gestión medioambiental.

El argumento económico que fundamenta este cambio de orientación, básicamente se centra en: los elevados costes de medidas de policía administrativa; la no incentivación para las empresas y la adopción de soluciones de «final de 
tubería»; la percepción de una injerencia o intervencionismo administrativo en base a demandas de grupos sociales ajenos al sistema empresarial y económico, que repercuten de manera negativa en la competitividad de las empresas. Como ha señalado García Ferrando (1994), «El stock de instrumentos heredados del pasado para coordinar las conductas de los distintos actores de las sociedades complejas aparecen necesitados también de un cambio controlado o experimental. La mera regulación, basada en la capacidad imperativa de la Administración, carece, por lo general del grado de afinamiento requerido para una acción eficaz, no cuenta con la información completa o sólo exhibe una legitimidad formal de carácter universal (...), redundando frecuentemente en desincentivar conductas voluntarias por encima de la norma o estándar fijado, además de resultar de difícil aplicación y seguimiento, por las dificultades de inspeccionar y sancionar a los potenciales infractores» (1994:29).

De hecho, la tendencia señala la aparición de un campo diverso que queda reflejado en la puesta en marcha de acuerdos voluntarios, y en el conjunto de disposiciones relativas a la iniciativa particular de carácter voluntario (mayoritariamente), como son el ecoetiquetaje, los sistemas de gestión y ecoauditoría. Con ello, se transfiere el protagonismo a las unidades productivas (empresas), reconociéndoles como los nuevos y necesarios sujetos activos de la política medioambiental. Gana entonces terreno un ámbito básicamente configurado como contractual, tanto en el ámbito de las relaciones empresa administración, como el diseñado como método de gestión medioambiental dentro de las empresas y que contempla en diversidad de grado la participación de los trabajadores y de sus representantes (Lerma, I., y García, E., 1997b). Así pues, podemos también plasmar dicho nuevo potencial escenario, donde se ha articulado una zona de consenso en la dinámica de las relaciones laborales (ver cuadro 6)

Dicho escenario, ejemplifica por una parte una cierta divergencia de intereses, que permite a los actores sociales vehiculizar sus discursos y dar satisfacción a nuevas demandas.

Junto a ello, ejemplifica el surgimiento de instrumentos institucionales para absorber en su juego de intereses la problemática ecológica y darle respuesta en su ámbito concreto de aplicación. No obstante, esa zona de consenso también permite perpetuar una dinámica que denota un cierto pacto implícito de productividad que liga las estrategias medioambientales de los actores, fijando por un lado las distancias entre ellos y por otro la disociación entre sus respectivos discursos y actitudes (Riechman, J., y Fernández, F., 1998; Recio, A. (1999). Estrategias que habida cuenta de su evolución, señalan una cautela y valoración excesiva de los costes que supondría para sus organizaciones ampliar el contenido medioambiental de las relaciones laborales. 
Cuadro 6

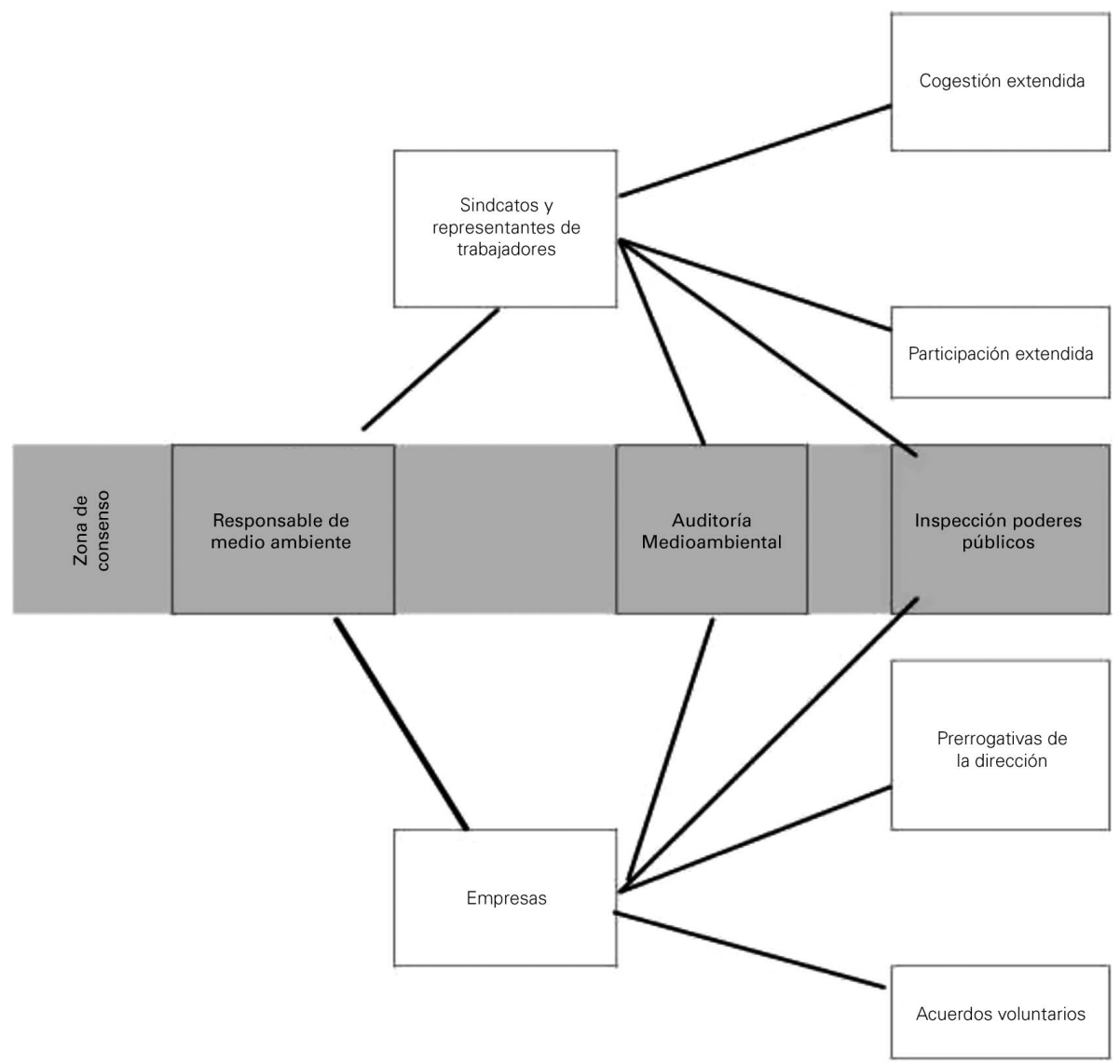

\section{Una propuesta de contenidos: un modelo para armar}

Dada la multidimensionalidad del objeto de análisis de referencia, recogido en el punto anterior, todo parece señalar que su adecuada traslación al curricula formativo de los Graduados en RRLL-RRHH, en cuanto a adquisición de competencias y comprensión de contenidos, debe estar presidido por la multidisciplinariedad como elemento básico para su articulación didáctica y docente. La propuesta de objetivos de aprendizaje y contenidos que se sigue, parafraseando a Julio Cortázar, supone un modelo para armar según las especificidades de cada plan de estudios sobre la base de su organización y metodología docente, de la existencia de seminarios o no, de actividades complementarias, o incluso, de su consideración estructurada en una materia optativa participada desde diversas 
áreas de conocimiento. Su formulación, pretende incorporar aquellos núcleos temáticos de relevancia para los Graduados en relación a su formación y capacitación profesional.

\section{A) Núcleo temático: desarrollo, crecimiento económico y medio ambiente}

\section{Objetivos de aprendizaje}

- Presentar y conocer el estado actual de las bases naturales de la vida humana.

_ Evaluar los efectos del desarrollo sobre el entorno ecológico y los sistemas sociales.

- Conocer las diferentes propuestas que se han realizado para hacer frente al problema de la cuestión ecológica y sus implicaciones sociales, así como las diferentes respuestas institucionales.

\section{Contenidos}

- Perfil y características de la crisis ecológica:

- Introducción: Sistemas naturales-sistemas sociales.

- Perfil, características y causas de la crisis ecológica actual. Modelo de desarrollo, crecimiento económico e impacto ambiental.

- Dimensiones de la crisis ecológica. El cambio ambiental global: Indicadores básicos.

—El desarrollo sostenible:

- El desarrollo sostenible, un dialogo de discursos: «de los límites al crecimiento» al «crecimiento de los límites».

- El dilema de la sostenibilidad: dimensiones y criterios de sustentabilidad.

- La respuesta de las instituciones políticas, económicas y sociales.

B) Nucleo temático: el marco normativo medioambiental y laboral: conexiones y proyección

\section{Objetivos de aprendizaje}

- Analizar las características y problemática de la regulación e instrumentación jurídica del derecho al medio ambiente en el marco de los derechos de ciudadanía o derechos de nueva generación.

- Presentar las tendencias en la evolución del derecho ambiental con relación a los cambios geopolíticos y su vinculación con los programas políticos e institucionales. Conocer los programas y la normativa ambiental comunitaria, centrándonos en aquella normativa de relevancia. 


\footnotetext{
LA INCLUSIÓN DE LA "CUESTIÓN ECOLÓGICA» EN EL CURRÍCULO

De los/as graduados/as en Relaciones Laborales y Recursos Humanos
}

- Presentar el marco normativo ambiental español, en sus diferentes ordenamientos, su grado de aplicación, niveles competenciales y responsabilidades derivadas.

- Estudiar la viabilidad de la extensión del contenido de las relaciones laborales hacia la atención del medio ambiente interno y externo de trabajo, a través del marco normativo laboral y contractual.

\section{Contenidos}

— La regulación medioambiental:

- ¿Derecho del medio ambiente o derecho al medio ambiente?. El derecho al medio ambiente como derecho de tercera generación. La configuración constitucional del Derecho al Medio Ambiente.

- El derecho ambiental internacional en el contexto de la globalización y mundialización de la economía. Los caracteres del derecho comunitario ambiental: la política comunitaria medioambiental; los nuevos instrumentos para la reconversión ecológica de las empresas.

- La privatización del conflicto ambiental por el ordenamiento civil: privatización del medio ambiente y tendencia a la negociación. La función simbólica del Derecho penal en materia medioambiental.

— Relaciones laborales y medio ambiente:

- Relaciones laborales y medio ambiente.

- Medio ambiente y ordenamiento laboral: los puntos de convergencia y las fricciones entre ecología y derecho laboral: Las instituciones laborales con proyección ecológica.

- Salud laboral, trabajo y medio ambiente. La viabilidad de la consideración del medio ambiente a través de la salud laboral.

\section{C) Núcleo temático: gestión ambiental de la empresa}

\section{Objetivos de aprendizaje}

- Conocer las teorías y modelos de organización de empresas tendentes a formular mecanismos integradores de la variable medioambiental, incorporando principios de sostenibilidad.

- Conocer los imperativos medioambientales para las empresas.

- Conocer los diferentes sistemas e instrumentos de gestión ambiental aplicados a la empresa y desarrollar modelos básicos de organización de empresas incorporando la temática medioambiental. 


\section{Contenidos}

— Empresa y medio ambiente:

- Medio ambiente y teorías de la organización.

- La empresa ecogestionada: motivaciones, cambios en las políticas empresariales.

- La ecoeficiencia: dimensiones.

- Estrategia ambiental de la empresa: etapas, posicionamiento y planificación estratégica ambiental.

— Sistema e instrumentos de gestión ambiental:

- Sistemas de gestión y diseño ambiental.

- Evaluación de impacto.

- Los elementos del SGMA.La documentación del sistema.

- Los RRHH en la gestión ambiental de la empresa.

- Sistemas integrados de gestión de la prevención, de la calidad y de la gestión ambiental.

D) Núcleo temático: los actores sociales sociales ante la cuestión ambiental: culturas, demandas y prácticas.

\section{Objetivos de aprendizaje}

- Conocer los diferentes puntos de vista de los actores sociales respecto a la cuestión ecológica y sus iniciativas.

- Presentar y analizar las diferentes propuestas de demandas de los actores sociales frente a la administración y entre ellos.

- Exponer y considerar, la influencia del movimiento ecologista, del cambio de valores y de las nuevas demandas ciudadanas.

- Significar aquellas prácticas institucionales, llevadas a cabo por los actores sociales así como determinar su orientación y contenidos.

- Valorar los acuerdos establecidos y considerar su grado de utilidad como elemento de corrección de prácticas perjudiciales para el medio ambiente.

\section{Contenidos}

- Posición de los actores sociales ante la crisis ecológica:

- Primeras consideraciones de la problemática medioambiental: La experiencia reivindicativa del movimiento obrero y sindical para dignificar el medio ambiente laboral.

- Actitudes sindicales: La crisis ecológica, su comprensión y programas de acción. La interrelación del movimiento sindical y del movimiento ecologista. 


\footnotetext{
LA INCLUSIÓN DE LA "CUESTIÓN ECOLÓGICA» EN EL CURRÍCULO

de los/as graduados/as en Relaciones Laborales y Recursos Humanos
}

- La consideración de la problemática ecológica por las asociaciones empresariales.

- Actitudes de las asociaciones empresariales: programas, demandas y necesidades para la reconversión ecológica de las empresas.

- Negociación colectiva y medio ambiente:

- Las prácticas de los actores: Consideración del medio ambiente en la negociación colectiva, su tratamiento a través de la concertación social y de la mesoconcertación. Contenidos, aplicación y grado de cumplimiento.

- Las prácticas de los actores: Consideración del medio ambiente en la negociación colectiva, su tratamiento a través de los convenios colectivos. Contenidos, aplicación y grado de cumplimiento.

- Otros contenidos de negociación: La potencialidad del empleo verde y sus limitaciones. Una perspectiva dual sobre la relación entre ecologización de la economía y generación de empleo.

\section{E) Núcleo temático: desarrollo territorial y medio ambiente}

\section{Objetivos de aprendizaje}

- Conocer la los instrumentos para la integración de la variable medioambiental en las políticas socioeconómicas de desarrollo territorial.

- Conocer los procedimientos operativos para implementar una política medioambiental local.

\section{Contenidos}

— Desarrollo local y sostenibilidad:

- Características de un modelo de desarrollo territorial sostenible.

- Políticas Públicas y gestión ambiental desde la escala regional-local.

- Agenda 21 Local: objetivos y proceso de implantación.

- La participación ciudadana.

- Análisis de buenas prácticas.

\section{Bibliografía}

Alonso, L.E. (1999). Trabajo y ciudadanía. Estudios sobre la crisis de la sociedad salarial, Madrid, Trotta.

Alonso, L.E. (2005). La era del consumo, Madrid, Siglo XXI.

Alonso, L.E. (2006). "A Post-Fordist Consumption Norm? Social Fragmentation, Individualization and New Inequalities», en Alonso, L.E. y Martínez, M. (ed.). Employment Relations in a Changing Society. Assesing the Post-Fordist Paradigm, Basingstoke: Palgrave, Macmillan, pp. 153-168. 
Aguilera, F.; Alcántara, V. (comp.) (1994). De la Economía Ambiental a la Economía Ecológica, Barcelona, FUHEM-Icaria.

Altvater, E. (1994). El precio del bienestar. Expolio del medio ambiente y nuevo (des)orden ecológico, Valencia, Alfons El Magnànim-IVEI.

Altvater, E. (2003). La teoria marxista hoy, problemas y perspectivas ¿Existe un marxismo ecológico hoy? Disponible en http://www. bibliotecavirtual.clacso.org.ar.

Altvater, E. (2009). "La Ecología desde una óptica marxista», curso Ecología política en el capitalismo contemporáneo, Programa Latinoamericano de Educación a Distancia, Centro Cultural de la Cooperación Floreal Gorini, Buenos Aires.

Banco Mundial (2008), "Informe sobre el desarrollo mundial 2008», En foco B: Biocombustibles: la promesa y los riesgos. Disponible en http://siteresources.worldbank.org/ INTIDM2008INSPA/Resources/En-foco-B.pdf.

Barcellona, P. (1995). El individualismo propietario. Madrid, Trotta.

FAO (2008). Declaración de la Conferencia de Alto Nivel sobre la Seguridad Alimentaria Mundial: los desafíos del cambio climático y la bioenergía, 3-5 de junio de 2008, Roma. Disponible en http://www.fao.org/filead-min/user_upload/foodclimate/HLCdocs/ declaration-S.pdf

Daly, H., Schütze, C., Beck, U., Dahl, J. (1997). "Crisis ecológica y sociedad», en Alzira, Germanía. Dolz, J. (1990). «Aproximación a la dimensión laboral de la protección del medio ambiente en Europa». Revista Jurídica La ley. Tomo I.

Duclos, D. (1991). Les industriels et les risques pour l'environnment. París, L'Harmattan.

FAO (2009). El estado de los mercados de productos básicos agrícolas 2009. Disponible en http://www.fao.org/docrep/ 012/i0854s/i0854s00.htm.

Folch, R. (2011). La quimera de creixer, Barcelona, La Magrana.

Friends of the Earth (2009). A dangerous obsesión. Disponible en http://www.foe. co.uk/resource/reports/dangerous_obsession.pdf.

Galeano, E.(1996). "La ecología en el marco de la impunidad», en VV.AA.: Ecología solidaria. Madrid, Trotta.

García, E. (1995a). El trampoli faustic: ciencia, mite i poder en el desenvolupament sostenible, Alzira, Germania.

García, E. (1995b). «Notas sobre desarrollo sustentable y propósito consciente», en Ecología politica, n. ${ }^{\circ} 10$.

García, E.-La Roca, F./Hildebrandt, E. y Külhleis, D. (1996). «Flexible employment practices and working time patterns: The potential for and limits of ecological impact", en Transfer, European Review of Labour and Research, vol 2, n. 3.

García, E. (2004). Medio ambiente y sociedad. La civilización industrial y los límites del planeta, Madrid, Alianza.

García, M. y Pardo, R. (1994). Ecología, relaciones industriales y empresa, Bilbao, BBV.

Georgescu-Roegen, N. (1975). "Energía y mitos económicos», en El Trimestre Económico, Vol. XLII, N.o 168,

Goodland, R., Daly, H., El Serafy, S., Von Droste, B. (1997). Medio ambiente y desarrollo sostenible. Más allá del Informe Brundtland, Madrid, Trotta.

Gorz, A. (1988). Métamorphoses du travail, Paris, Galilée.

Gorz, A. 1991). Capitalisme Socialisme Écologie, Paris, Galilée.

Global Humanitarian Forum (2009). Human Impact Report: Climate Change - The Anatomy of $A$ Silent Crisis. Disponible en http://www.ghfgeneva.org/Portals/0/pdfs/ human_impact_report.pdf. 
Gould, K.A.; Schnaiberg, A.; Weinberg, A. (1996). Local environmental struggles: citizen activism in the treadmill of production, Nueva York, Cambridge University Press.

Guattari, F. (1990). Las tres ecologias. Valencia, Pre-Textos.

Hadeler, K.P. (1982). Matemáticas para biólogos, Barcelona, Reverté.

Hildebrandt, E.-Fondation Européenne pour l'Amélioration des Conditions de VIE ET de Travail (1992). Relations professionelles et environnement dans la Communauté Européenne, Luxemburgo, Oficina de Publicaciones de las Comunidades Europeas.

Hildebrandt, E. y Schmidt, E. (1994). Industrial Relations and the environnemental Protection in Europe. Dublín, Fundación Europea para la Mejora de las Condiciones de Vida y Trabajo.

Hildebrandt, E. y OAtes, E. (1997). Work, employment and environment: Quality and quantity of work in the environmental labour market and its regulation, Berlin, Wissenschaftszentrum fur Sozialforschung.

Hirsch, F. (1976). Social limitation to growth. Cambridge, Harvard University Press.

International Atomic Energy Agency (2008). IAEA Annual Report for 2008, The year in review. Disponible en http://www.iaea.org/ Publications/Reports/Anrep2008/ review.pdf.

Jiménez, L.M. (2001). Medio ambiente y desarrollo alternativo, Madrid, Sistema.

LEFF, E. (2005). «La geopolítica de la biodiversidad y el desarrollo sustentable», Revista del Observatorio Social de América Latina, No. 17, Buenos Aires.

La Roca, F. y Sánchez, A. (1996). Economía crítica. Trabajo y medio ambiente. Valencia, FEIS-Univ. València.

Lerma, I. y García, E. (1994). «Medio ambiente y relaciones industriales: un análisis en el contexto europeo». en García Ferrando, M. Pardo Avellaneda, R.: Ecología, relaciones industriales y empresa. Bilbao, BBV.

Lerma, I., García, E. (1997a). Relaciones laborales y medio ambiente. Alzira, Germanía.

LERMA, I. y GARCÍA, E. (1997b). La participación de los trabajadores y trabajadoras en la gestión medioambiental de las empresas. Alzira, Germanía.

Lipietz, A. (1988). «El mundo del Postfordismo», en Ensayos de Economía n.o 12, vol. 7.

Martínez-Alier, J.; Schlüpmann, K. (1992). La ecología y la economía, Madrid, Fondo de Cultura Económica de España.

Martínez Alier, J. (1995). De la economía ecológica al ecologismo popular, Barcelona, Icaria,

Martínez Alier, J. (2005). Los conflictos ecológico-distributivos y los indicadores de sustentabilidad. Disponible en: http://www.rebelion.org/noticia.php?id=22206.

Marx, K. (1982). El Capital. Crítica de la economía política, México, FCE.

Naredo, J. (1993). Hacia una ciencia de los recursos naturales, Madrid, Siglo XXI.

OAtes, A. y Gergory, D. (1993) (eds.). Industrial relations and environment: Ten countries under the microscope, Dublín, Fundacion Europea para la Mejora de las Condiciones de vida y Trabajo, (2 vols.).

O’Connor, J. (1990). "Las condiciones de producción. Por un marxismo ecológico», en Ecología política, n. ${ }^{\circ} 1$.

O'Connor, J. (2001). Causas naturales. Ensayos de marxismo ecológico, México, Siglo XXI.

Ovejero, F. (1989). Intereses de todos, acciones de cada uno, Madrid, Siglo XXI.

Recio, A. (1999). «Sindicatos, globalización económica y crisis ambiental» en Papers, n. ${ }^{\circ} 58$.

Renner, M. (1994). El empleo en una economía sostenible. Bilbao, Bakeaz. 
Riechmann, J. y Fernández, F. (1998). Trabajar sin destruir. Trabajadores, sindicatos y ecologismo, Barcelona, HOAC.

Riechmann, J. (1998) (coord.). Necesitar, desear, vivir. Sobre necesidades humanas, desarrollo humano, crecimiento económico y sustentabilidad. Madrid, Los libros de la Catarata.

Riechman, J. (1998). "Empleo en la transición hacia una economía sustentable: posibilidades y límites», en Arxius de Sociología n. ${ }^{\circ} 2$.

Riechmann, J. (2006) «Termodinámica: ¿Por qué los muertos no resucitan y el reciclaje perfecto es imposible?», en Fernández, F., Riechmann, J. Ni tribunos: ideas y materiales para un programa ecosocialista, Madrid, Siglo XXI.

Rodríguez, M. (1995). «Trabajo y medio ambiente» en La Ley, Tomo I.

Sabbatella, I. (2008). "Capital y naturaleza: crisis, desigualdad y conflictos ambientales», ponencia presentada en las II Jornadas de Economía Política, noviembre de 2008, Universidad de General Sarmiento, Buenos Aires.

Sabbatella, I. (2009). «Ecological crisis and nature subsumed to capital», Íconos. Revista de Ciencias Sociales, núm. 36, Quito, enero de 2010, pp. 69-80.

SACHS, W. (2000). Globalization and Sustainability: an essay. Berlin, Heinrich Böll Foundation.

Sachs, W.; Santarius, T. et al. (2007). Fair Future. Resource Conflicts, Security, and Global Justice, London, Zed Books, 2007.

Stern, N., (2006). Review on the Economics of Climate Change. Chapter 2 Economics Ethics and Climate Change. Disponible en http://www.hm-treasury. gov. uk/d/Chapter 2 Economics Ethics and Climate Change.pdf

Schaniberg, A. (1980). The environment: from surplus to scarcity, New York, Oxford University Press.

World Wide Fund for Nature (WWF), 2008 y sucesivos informes, Planeta Vivo. Disponible en http://www. wwf.es/index.cfm, 